\title{
Epithelioid Hemangioendothelioma of the Liver: About A Case and Review of the Literature
}

\author{
R. Chaouachi ${ }^{*}$ I. Ben moula, Pr. Chikaoui, Pr. kacimi, Pr. Touil, Pr. A Siwane, Pr. Tabarkh
}

Emergency Radiology Chu Ibn Rochd Casablanca Morocco

DOI: $10.36347 /$ simcr.2020.v08i07.002

| Received: 30.06 .2020 | Accepted: 08.07.2020 | Published: 12.07.2020

*Corresponding author: Chaoauchi Rabeb

Abstract

Epithelioid hemangioendothelioma of the liver is a rare primary malignant tumor of vascular origin. It most frequently occurs in middle age adults and clinical findings are non-specific. Histological diagnosis may be difficult to achieve with lesions containing a large myxoid component, and immunohistochemical staining is required. A few imaging features may suggest the correct diagnosis, and radiologists should be familiar with these findings. We will illustrate the potential role of PET-CT at the time of initial presentation. Prognosis is variable and optimal management is based on a case by case analysis, ranging from clinical and imaging follow-up to liver transplantation.

Keywords: Liver, Malignant tumor, US, CT, MRI.

Copyright @ 2020: This is an open-access article distributed under the terms of the Creative Commons Attribution license which permits unrestricted use, distribution, and reproduction in any medium for non-commercial use (NonCommercial, or CC-BY-NC) provided the original author and source are credited.

\section{INTRODUCTION}

A young 25-year-old patient with no specific medical history presented to the emergency room with predominant abdominal pain in the right hypochondrium. On interrogation, there was deterioration in the general condition associated with a loss of $3 \mathrm{~kg}$. There was no jaundice, transit disorders or peripheral adenomegaly on palpation. The biological assessment found 12,000 GB, a CRP at $19 \mathrm{mg} / \mathrm{ml}$ and the hepatic assessment was strictly normal. An abdominal ultrasound was performed and showed multifocal hypoechoic liver damage. It was completed by an abdominal-pelvic scanner with injection of iodinated contrast agent. the liver presented a confluent lesion of the right liver (figure 1) with a homolateral atrophy associated with a contralateral compensatory hypertrophy and in particular of segment I. A hepatic MRI and a PET-CT with 18FDG were therefore carried out. On the PET-CT scan (fig. 2); the liver lesions appeared hypermetabolic. On MRI (Fig. 3), the lesions appeared in $\mathrm{T} 1$ hyposignal without intralesional fatty content and in heterogeneous central T2 hypersignal with a target appearance. After injection of gadolinium chelate, there was an uptake of contrast in the portal phase, which intensified in the late period, reflecting the significant associated fibrosis. An echo-guided biopsy of liver damage confirmed the diagnosis of epithelioid hemangioendothelioma. After multidisciplinary consultation, it was decided to monitor the patient regularly without immediate radical treatment. At 6 months from the initial diagnosis, the lesions appeared stable in number and size

\section{DISCUSSION}

Epithelioid hemangioendothelioma is a rare primary liver tumor of mesenchymal origin. It usually affects middle-aged subjects (average age: 45) with a slight predominance of women (sex ratio: $3 / 2$ ) with no risk factors identified.

The circumstances of discovery of epithelioid hemangioendothelioma are very variable and nonspecific, most often fortuitous. Exceptionally, it is revealed by a Budd-Chiari syndrome linked to venous invasion, a hemoperitoneum by intraperitoneal rupture or hepatocellular insufficiency by replacement of the healthy liver. From a macroscopic point of view, the lesions have a multifocal character affecting the two hepatic lobes. They are in the form of nodules of variable size $(0.5$ to $12 \mathrm{~cm})$ tending to converge. The topography of the lesions is rather peripheral with contiguous capsular extension. The capsular retraction is secondary to the significant fibrosis of the periphery of the nodular lesions. The spared and healthy segments show a compensatory hypertrophy [1].

Microscopically,

epithelioid hemangioendothelioma is characterized by a large myxoid fibrous stroma. The key point in pathology diagnosis is based on immunohistochemical labeling: 
the epithelioid hemangioendothelioma appears positive for at least one endothelial marker (CD31, CD34 and factor VIII Rag) and negative for epithelial markers [2].

Ultrasound is often the first-line examination and is supplemented by a CT or MRI examination of the liver before the finding of numerous hepatic lesions of variable echogenicity [3]. These allow finding some radiological signs suggestive of the pathological diagnosis such as capsular retraction, peripheral involvement, the possible presence of calcifications within the lesions and the alternation of atrophy of the tumor liver and hypertrophy of the non-tumor liver. After injection, the hypodense lesions can take on a target appearance with moderate peripheral contrast enhancement. On late acquisitions, the nodules become isodense. Sometimes we find a portal or hepatic vessel coming into contact with the lesion, an aspect described under the name of "Lollipop sign" by the Anglo-Saxons [4].

However, MRI is the most effective radiological examination to highlight peripheral lesional fibrosis, resulting in delayed and retentional contrast enhancement [5]. The target appearance on T2weighted sequences is also very characteristic and should be sought.

The appearance of epithelioid hemangioendothelioma in its hepatic form on 18FDG PET-CT has only been reported in two observations. This rare tumor appears hypermetabolic, PET can detect possible other lesions at a distance from its whole body exploration [6]. The progression of the tumor is unpredictable going from the indolent form with slow growth without general repercussions to a rapidly progressive and immediately darker evolution towards hepatocellular insufficiency. Survival without curative treatment is possible and estimated at $30 \%$ at 5 years.

The therapeutic management of hemangioendothelioma is adapted according to the presentation of the hepatic injury, the symptomatology of the patient and the speed of evolution. It varies from simple radiological monitoring to surgical excision of the lesions or to liver transplantation [7].

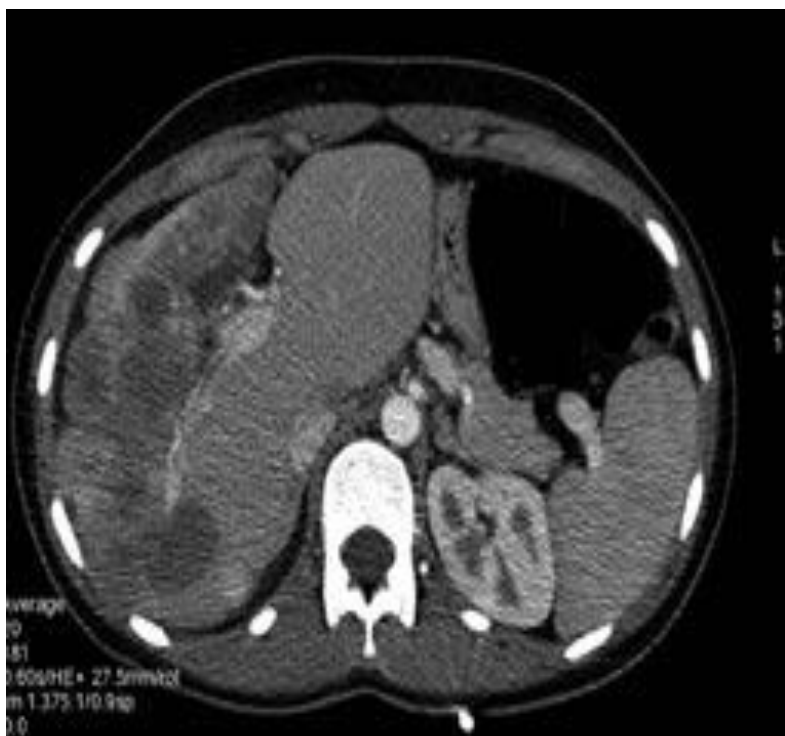

Fig-1: Abdominopelvic CT showing the confluent liver lesions discreetly enhanced at the periphey as well as the capsular retraction and compensatory enlargement of the healthy liver

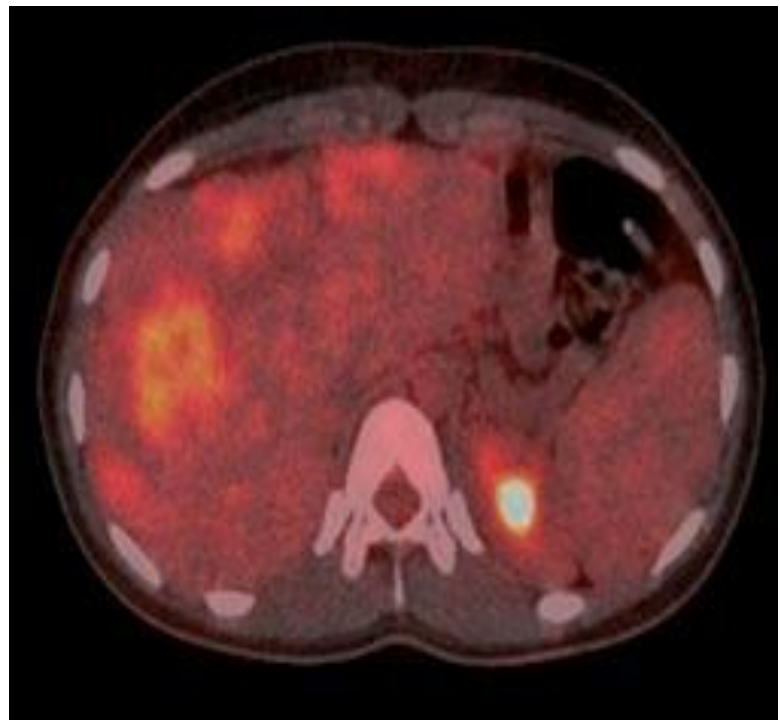

Fig-2: 18FDG PET-CT revealing the hyperfixing nature of the lesions

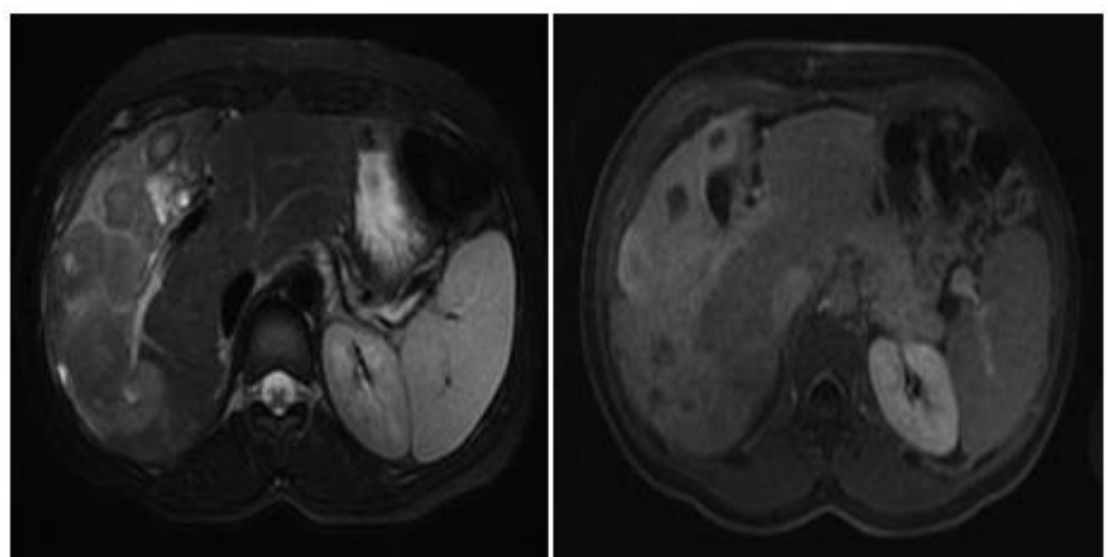

Fig-3: Liver MRI. Lesions have a target appearance on T2-weighted images (1st image) with significant late contrast enhancement due to extensive fibrosis (2nd image) 


\section{REFERENCE}

1. Miller WJ, Dodd GD 3rd, Federle MP, Baron RL. Epithelioid hemangioendothelioma of the liver: imaging findings with pathologic correlation. AJR Am J Roentgenol. 1992; 159:53-7.

2. Makhlouf HR, Ishak KG, Goodman ZD. Epithelioid hemangioendothelioma of the liver: a clinicopathologic study of 137 cases. Cancer. 1999; 85:562-82.

3. Lyburn ID, Torreggiani WC, Harris AC. Hepatic epithelioid hemangioendothelioma: sonographic, CT, and MR imaging appearances. AJR Am J Roentgenol. 2003; 180:1359-64.
4. Alomari AI. The lollipop sign: a new crosssectional sign of hepatic epithelioid hemangioendothelioma. Eur J Radiol. 2006; 59:460-4.

5. Van Beers B, Roche A, Mathieu D, Menu Y. Epithelioid hemangioendothelioma of the liver: MR and CT findings. J Comput Assist Tomogr. 1992; 16:420-4.

6. Nguyen BD. Epithelioid hemangioendothelioma of the liver with F-18 FDG PET imaging. Clin Nucl Med. 2004; 29:828-30.

7. Ben-Haim M, Roayaie S, Ye MQ, Thung SN. Hepatic epithelioid hemangioendothelioma: resection or transplantation, which and when? Liver Transpl Surg. 1999; 5:526-31. 